\title{
Late to bed, late to rise, could be cardiometabolically unwise
}

Inconsistency in weekly sleep patterns is associated with adverse cardiovascular and metabolic variables, according to results published in The Journal of Clinical Endocrinology \& Metabolism. Harmonising sleep behaviour on days with and without work has the potential to reduce the incidence of type 2 diabetes mellitus (T2DM) and atherosclerotic cardiovascular disease (CVD).

Poor quality and short duration of sleep are known to be associated with increased risks of the metabolic syndrome, T2DM and CVD. Shift work, which results in chronic circadian misalignment, is also associated with these disorders. However, the effects on these risks of day-to-day circadian disruption in healthy adults with 'normal' work patterns are not known. The difference between sleep timing on work days and 'free' days is known as 'social jetlag. Sleep timing is also influenced by chronotype, which describes patterns of preferred bedtimes and subjective assessment of peak alertness. Evening chronotype and social jetlag have both been linked to adverse health measures, but their precise associations with preclinical cardiometabolic alterations have not been defined.

Patricia Wong and colleagues have now examined the relationships between chronotype, social jetlag and cardiometabolic risk in 447 healthy adults (mean age 42.7 years, $53 \%$ female, $83 \%$ white) over the course of 7 days involving a total of $>25 \mathrm{~h}$ of daytime work for each participant. Chronotype was assessed with the Composite Scale of Morningness. Continuous actigraphy was used to determine sleep duration, timing and efficiency (the proportion of total rest time spent in sleep). Haemodynamic and metabolic variables and symptoms of depression were assessed at the beginning of the study, and health behaviours relating to cigarette smoking, alcohol intake, physical activity and food intake were recorded.

Social jetlag (the shift in the average midpoint of sleep from free days to work days) ranged from $-120 \mathrm{~min}$ to $180 \mathrm{~min}$. In $84.8 \%$ of individuals the midsleep was later on free days than on work days. Social jetlag was positively correlated with the tendency to have an evening chronotype (the preference for later sleep timing) With hierarchical regression analysis, controlling for demographics, sleep characteristics, health behaviours and depression, social jetlag was positively correlated with levels of triglycerides ( $\beta$ coefficient $=0.11, P=0.026)$, fasting insulin $(\beta=0.11, P=0.033)$, insulin resistance $(\beta=0.11, P=0.031)$, waist circumference $(\beta=0.15, P=0.002)$ and $\operatorname{BMI}(\beta=0.17, P=0.001)$, and was negatively correlated with levels of HDL cholesterol $(\beta=-0.09, P=0.036)$. The 'eveningness' of the chronotype was negatively correlated with HDL cholesterol $(\beta=-0.08, P=0.047)$.

Despite the observed correlations, these results might actually underestimate the association between social jetlag and cardiometabolic risk. Only $4.9 \%$ of these middle aged individuals met the threshold for evening chronotype (previously linked with depression, overweight, T2DM and hypertension), and the mean social jetlag was only $44 \mathrm{~min}$. Furthermore, associations with other relevant risk factors, such as postprandial glycaemia and food consumption in the evenings, were not determined.

This cross-sectional study has highlighted the cardiometabolic risks associated with behaviours that can disrupt the intrinsic circadian rhythms of physiological processes such as glucose metabolism and the regulation of body temperature and blood pressure. Prospective investigations are now required, with interventions to determine whether the reduction of social jetlag can normalize these cardiometabolic variables.

\section{Robert Phillips}

ORIGINAL ARTICLE Wong, P. M. et al. Social jetlag, chronotype, and cardiometabolic risk. J. Clin. Endocrinol. Metab. http://dx.doi.org/ 10.1210/jc.2015-2923

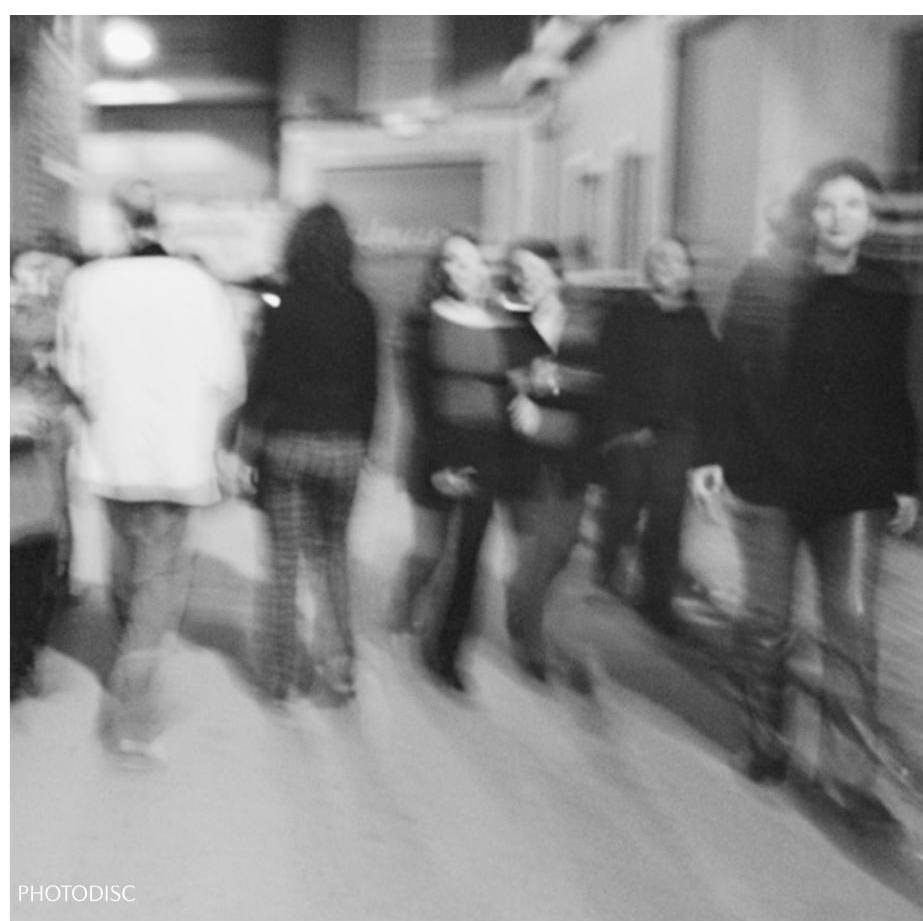

\section{these results might actually underestimate the association between social jetlag and cardio- metabolic risk

